Pacific Journal of Mathematics

MULTIPLICATIVITY OF THE LOCAL HILBERT SYMBOL 


\section{MULTIPLICATIVITY OF THE LOCAL HILBERT SYMBOL}

\section{RONALD JACOBOWITZ}

1. Introduction. Let $F$ be a commutative field of characteristic not 2 , complete under a discrete, non-archimedean valuation | |, with finite residue class field-such a field is often called local-for example, the field of ordinary $p$-adic numbers. For nonzero elements $\alpha, \beta$ of $F$, the Hilbert symbol $(\alpha, \beta)$ is defined to be +1 or -1 according as the equation $\alpha x^{2}+\beta y^{2}=1$ is or is not solvable in $F$. It has such obvious properties as $(\beta, \alpha)=(\alpha, \beta),\left(\alpha, \beta \gamma^{2}\right)=(\alpha, \beta),(\alpha,-\alpha \beta)=(\alpha, \beta)$; and if at least one of $(\alpha, \beta),(\alpha, \gamma)$ is +1 , then

$$
(\alpha, \beta)(\alpha, \gamma)=(\alpha, \beta \gamma)
$$

as is easily seen by observing (whether or not $\alpha \in F^{2}$ )

(2) $(\alpha, \beta)=+1$ if and only if $\beta \in N_{E / F} E$, where $E=F\left(\alpha^{1 / 2}\right)$.

These properties are true even without the assumption that $F$ is local; under that assumption, however, the multiplicative property (1) is always true, i.e., $(\alpha, \beta)=(\alpha, \gamma)=-1 \Rightarrow(\alpha, \beta \gamma)=+1$. In [3], Example 63:12, O'Meara derives this result from the study of local quaternion algebras by applying Wedderburn structure theory to tensor products of such algebras. The point of the present paper is to give a direct proof, using only the most elementary facts about non-archimedean valuations (such as found in [3], Chap. I). Specifically, we shall prove the so-called "second inequality of local class field theory" for quadratic extensions, i.e., $\left(F^{*}: N_{E / F} E^{*}\right) \leqq 2$, where $E$ is an arbitrary quadratic extension of $F$, and $F^{*}$ and $E^{*}$ denote, respectively, the nonzero elements of $F$ and $E$; the required property (1) will then follow immediately, because of (2).

2. Proof of the second inequality. Since the ramification number of $E / F$ is at most 2([3], Proposition 13:6), an obvious computation shows that it suffices to prove the

PROPOSITION.

$$
\begin{cases}\left(\mathfrak{H}: N_{E / F} \mathfrak{H}\right)=1 & \text { if } E / F \text { is unramified } \\ \left(\mathfrak{H}: N_{E / F} \mathfrak{U}\right) \leqq 2 & \text { if } E / F \text { is ramified }\end{cases}
$$

where $\mathfrak{t}=\{\varepsilon \in F|| \varepsilon \mid=1\}$ and $\mathfrak{U}=\{a \in E|| a \mid=1\}$, the units of $F$ and $E$, respectively. 
The proof of the Proposition will be broken up into several steps. First, let $\pi$ denote a generic prime element (to be specified later) for $F$, and for each positive rational integer $n$, define $\mathfrak{t}_{n}=\left\{\varepsilon \in \mathfrak{u} \mid \varepsilon \equiv 1\left(\bmod \pi^{n}\right)\right\}$, a subgroup of $\mathfrak{t}$. Also define the nonnegative integer $e$ by $|\pi|^{e}=|2|$; thus $e=0$ in the non-dyadic case $(|2|=1), e>0$ in the dyadic case $(|2|<1)$. We obviously have $\mathfrak{u} \supseteqq \mathfrak{u}_{1} \supseteqq \mathfrak{u}_{2} \supseteqq \ldots \supseteqq \mathfrak{u}_{2 \varepsilon} \supseteqq \mathfrak{u}_{2 e+1} \supseteqq \ldots$ Furthermore, by Hensel's lemma ([3], Theorem 63:1), $\mathfrak{u}_{2 e+1} \subseteq \mathfrak{u}^{2} \subseteq N \mathfrak{U}$ (notation: $N=N_{E / F}$ ), thus we can write $\mathfrak{H} \supseteqq \mathfrak{H}_{1} N \mathfrak{U} \supseteqq \mathfrak{H}_{2} N \mathfrak{U} \supseteqq \cdots \supseteqq$ $\mathfrak{H}_{2 e} N \mathfrak{U} \supseteqq \mathfrak{H}_{2 e+1} N \mathfrak{U}=N \mathfrak{U}$; since group-indices multiply, we therefore have

LEMMA 1. $(\mathfrak{u}: N \mathfrak{U})=$

$$
\left(\mathfrak{u}: \mathfrak{H}_{1} N \mathfrak{U}\right)\left(\mathfrak{u}_{1} N \mathfrak{U}: \mathfrak{u}_{2} N \mathfrak{U}\right) \cdots\left(\mathfrak{U}_{2 e-1} N \mathfrak{U}: \mathfrak{u}_{2 e} N \mathfrak{U}\right)\left(\mathfrak{u}_{2 e} N \mathfrak{U}: \mathfrak{u}_{2 e+1} N \mathfrak{U}\right) .
$$

We next refer to [2], $\S 5$, for a classification of the several types of extensions $E / F$, namely:

Non-dyadic: Unramified if $E=F\left(\theta^{1 / 2}\right)$ with $|\theta|=1$

Ramified if $E=F\left(\pi^{1 / 2}\right)$

Dyadic: $\quad$ Unramified if $E=F\left((1+4 \delta)^{1 / 2}\right)$ with $|\delta|=1$

Ramified (" $R$-P") if $E=F\left(\pi^{1 / 2}\right)$

Ramified (" $R$-U”) if $E=F\left(\left(1+\pi^{2 k+1} \delta\right)^{1 / 2}\right) \quad$ with

$$
|\delta|=1 \text { and } 0 \leqq k \leqq e-1 \text {. }
$$

Here $\pi$, of course, denotes some particular prime element for $F$. In the case we are calling " $R$ - $U$ ", recall from [2], p. 454, that $p=$ $\left[1+\left(1+\pi^{2 k+1} \delta\right)^{1 / 2}\right] / \pi^{k}$ satisfies $N p=-\pi \delta$ and hence can (and shall) serve as prime element for $E$; and in " $R$ - $P$ ", we shall take $p=\pi^{1 / 2}$ as prime element for $E$. Let us also write $\mathfrak{D}=\{\alpha \in F|| \alpha \mid \leqq 1\}$, the "integers" of $F$.

LeMma 2.

$$
\begin{cases}\left(\mathfrak{u}: \mathfrak{u}_{1} N \mathfrak{U}\right)=1 & \text { in the unramified non-dyadic, and } \\ & \text { the three dyadic cases; } \\ \left(\mathfrak{u}: \mathfrak{u}_{1} N \mathfrak{U}\right) \leqq 2 & \text { in the ramified non-dyadic case } .\end{cases}
$$

Proof. The composite map $\mathfrak{u} \underset{\mathrm{CAN}}{\longrightarrow} \mathfrak{F}^{*} \underset{\mathrm{CAN}}{\longrightarrow} \mathfrak{F}^{*} / \mathfrak{F}^{* 2}$, $\mathfrak{F}$ denoting the residue class field of $\mathrm{F}$, is a multiplicative epimorphism with kernel $\mathfrak{u}_{\mathfrak{1}} \mathfrak{t}^{2}$, so $\left(\mathfrak{u}: \mathfrak{u}_{1} N \mathfrak{H}\right) \leqq\left(\mathfrak{H}: \mathfrak{u}_{1} \mathfrak{u}^{2}\right)=$ order of $\mathfrak{F}^{*} \mathfrak{F}^{* 2}$; since $\mathfrak{F}$ is finite, this order is 1 in the dyadic case, 2 in the non-dyadic. This proves the Lemma except in the unramified non-dyadic case, where we need a sharper estimate; however, in that case, we can apply Proposition 
62:1 of [3] (which shows that for any unit $\varepsilon$ of $F$, the congruence $\varepsilon x^{2}+\theta y^{2} \equiv 1(\bmod \pi)$ can be solved in $\left.\mathrm{o}\right)$ and Hensel's lemma to conclude that the Hilbert symbol $(\varepsilon, \theta)$ is equal to +1 for all $\varepsilon$ in $\mathfrak{u}$, hence $\mathfrak{u}=N \mathfrak{H}$.

Lemma 3. Suppose $E / F$ is dyadic. Then $\left(\mathfrak{u}_{n} N \mathfrak{U}: \mathfrak{u}_{n+1} N \mathfrak{U}\right)=1$ in the following cases: Unramified: $1 \leqq n \leqq 2 e$

$R-P: \quad 1 \leqq n \leqq 2 e-1$

$R-U: \quad 1 \leqq n \leqq 2(e-k)-2$ and $2(e-k) \leqq n \leqq 2 e$.

Proof. Our procedure will be, given $\varepsilon=1+\pi^{n} \alpha$ in $\mathfrak{t}_{n}$ (thus with $\alpha \in \mathfrak{D})$, to construct $a$ in $\mathfrak{U}$ with $\varepsilon \equiv N a\left(\bmod \pi^{n+1}\right)$, thus $\varepsilon / N a \in \mathfrak{H}_{n+1}$, thus $\varepsilon \in \mathfrak{H}_{n+1} N \mathfrak{U}$; this will show $\mathfrak{H}_{n} \subseteq \mathfrak{H}_{n+1} N \mathfrak{U}$, hence $\mathfrak{H}_{n} N \mathfrak{U}=\mathfrak{H}_{n+1} N \mathfrak{U}$. We consider five cases (note that II and V overlap, which simply means that either construction will work).

( I ) Unramified. Take $a=1+\pi^{n} \alpha\left(1+(1+4 \delta)^{1 / 2}\right) / 2$.

(II) $R-P$ or $R-U, n=2 r$ even, $1 \leqq r \leqq e-1$. Recalling that $\mathfrak{F}$ is finite of characteristic 2 , find $\beta \in \mathfrak{o}$ with $\beta^{2} \equiv \alpha(\bmod \pi)$, and take $a=1+\pi^{r} \beta$.

(III) $\quad R-P, \quad n=2 r+1$ odd, $0 \leqq r \leqq e-1$. Find $\beta \in \mathfrak{D}$ with $\beta^{2} \equiv-\alpha(\bmod \pi)$, and take $a=1+p \pi^{r} \beta$.

(IV) $R-U, n=2 r+1$ odd, $0 \leqq r \leqq e-k-2$. Find $\beta \in \mathfrak{D}$ with $\beta^{2} \equiv-\alpha / \delta(\bmod \pi)$, and take $a=1+p \pi^{r} \beta$.

( V ) $R-U, n \geqq 2(e-k)$. Take $a=1+p \pi^{n+k} \alpha / 2$. We check in each case that $a$ belongs to $\mathfrak{U}$ and $\varepsilon \equiv N a\left(\bmod \pi^{n+1}\right)$.

For the remaining two indices, we have

LEMMA 4. In $R-P$, $\left(\mathfrak{H}_{2 e} N \mathfrak{U}: \mathfrak{H}_{2 e+1} N \mathfrak{u}\right) \leqq 2$; in $R-U$, $\left(\mathfrak{H}_{2(e-k)-1} N \mathfrak{U}: \mathfrak{H}_{2(e-k)} N \mathfrak{U}\right) \leqq 2$.

Proof. The first of the two inequalities is easily disposed of by Proposition 63:4 of [3], which essentially states that $\left(\mathfrak{H}_{2 e}: \mathfrak{t}_{2 e+1}\right)=2$, so we turn to the second. Note that (for $\beta$ in $\mathfrak{o}) N\left(1+p \pi^{e-k-1} \beta\right.$ ) $=$ $1+\pi^{2(e-k)-1}\left(2 \pi^{-e} \beta-\delta \beta^{2}\right)$, and set $\mathfrak{R}=\left\{N\left(1+p \pi^{e-k-1} \beta\right) \mid \beta \in \mathfrak{p}\right\}$. Now we may assume $\mathfrak{H}_{2(e-k)-1}$ is not a subset of $\mathfrak{H}_{2(e-k)} N \mathfrak{M}$, and so can fix $\varepsilon_{0}=1+\pi^{2(e-k)-1} \alpha_{0}$ in $\mathfrak{H}_{2(e-k)-1}$ but not in $\mathfrak{H}_{2(e-k)} N \mathfrak{U}$, hence with $\varepsilon_{0} \notin \mathfrak{N}$, hence with $\alpha_{0}$ not of the form $2 \pi^{-e} \beta-\delta \beta^{2}$; then for any $\varepsilon=$ $1+\pi^{2(e-k)-1} \alpha$ in $\mathfrak{u}_{2(e-k)-1}$ but not in $\mathfrak{u}_{2(e-k)} N \mathfrak{U}$, we also have $\alpha$ not of 
the form $2 \pi^{-e} \beta-\delta \beta^{2}$, and since, reading modulo $\pi$, elements of the form $2 \pi^{-e} \beta-\delta \beta^{2}$ determine an additive subgroup of $\mathfrak{o}$ of index 2 , we can find $\beta_{1}$ in 0 with $\alpha+\alpha_{0} \equiv 2 \pi^{-e} \beta_{1}-\delta \beta_{1}^{2}(\bmod \pi)$, so that $\varepsilon \varepsilon_{0} \equiv$ $N\left(1+p \pi^{e-k-1} \beta_{1}\right)\left(\bmod \pi^{2(e-k)}\right)$, i.e., $\varepsilon \in \varepsilon_{0} \mathfrak{u}_{2(e-k)} N \mathfrak{u}$; thus the index $\left(\mathfrak{H}_{2(e-k)-1} N \mathfrak{U}: \mathfrak{H}_{2(e-k)} N \mathfrak{U}\right)$ is at most 2 .

q.e.d.

The proof of the Proposition, and thus the multiplicative property (1), now follows by combining the four Lemmas.

3. Concluding remarks. The "first inequality of local class field theory" states $\left(F^{*}: N E^{*}\right) \geqq 2$, and can also be proven directly-cf. [3], Propositions 63:13 and 63:13a. Its significance for us is that each of our index-inequalities in the Proposition and Lemmas 2 and 4 is now seen to be an equality.

As Durfee has shown in [1], the local isometry invariants for quadratic forms can easily be derived once our multiplicative property is known. Similarly, in the more modern, "geometric" treatment given in [3], $58, \S 63$, it is not difficult to reinterpret O'Meara's quaternion algebra $(\alpha, \beta)$ as a Hilbert symbol, tensor product $\otimes$ as ordinary multiplication, and algebra-similarity $\sim$ as equality; most of the arithmetic results of [3] then follow readily from the multiplicative property (1) and the Hasse theorem that any form in five variables is locally isotropic.

\section{REFERENCES}

1. W. H. Durfee, Quadratic forms over fields with a valuation, Bull. Amer. Math. Soc., 54 (1948), 338-351.

2. R. Jacobowitz, Hermitian forms over local fields, Amer. Jour. Math., 84 (1962), 441465.

3. O. T. O'Meara, Introduction to Quadratic Forms, Berlin, 1963.

The UNIVERSITY OF ARIZONA 


\section{PACIFIC JOURNAL OF MATHEMATICS}

\section{EDITORS}

Robert Osserman

Stanford University

Stanford, California

M. G. Arsove

University of Washington

Seattle 5 , Washington
J. DugundjI

University of Southern Califorma: Los Angeles 7, California

Lowell J. Paige

University of California

Los Angeles 24, California

\section{ASSOCIATE EDITORS}

E. F. BECKENBACH

B. H. NeumanN

F. WOLF

K. YosIDA

\section{SUPPORTING INSTITUTIONS}

UNIVERSITY OF BRITISH COLUMBIA

CALIFORNIA INSTITUTE OF TECHNOLOGY

UNIVERSITY OF CALIFORNIA

MONTANA STATE UNIVERSITY

UNIVERSITY OF NEVADA

NEW MEXICO STATE UNIVERSITY

OREGON STATE UNIVERSITY

UNIVERSITY OF OREGON

OSAKA UNIVERSITY

UNIVERSITY OF SOUTHERN CALIFORNIA
STANFORD UNIVERSITY

UNIVERSITY OF TOKYO

UNIVERSITY OF UTAH

WASHINGTON STATE UNIVERSITY

UNIVERSITY OF WASHINGTON

AMERICAN MATHEMATICAL SOCIETY CALIFORNIA RESEARCH CORPORATION SPACE TECHNOLOGY LABORATORIES NAVAL ORDNANCE TEST STATION 


\section{Pacific Journal of Mathematics}

\section{Vol. 14, No. 1 \\ May, 1964}

Richard Arens, Normal form for a Pfaffian .........................

Charles Vernon Coffman, Non-linear differential equations on cones in Banach

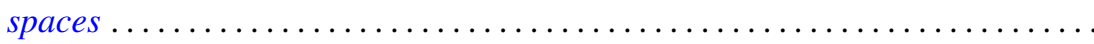

Ralph DeMarr, Order convergence in linear topological spaces ..............

Peter Larkin Duren, On the spectrum of a Toeplitz operator ................

Robert E. Edwards, Endomorphisms of function-spaces which leave stable all

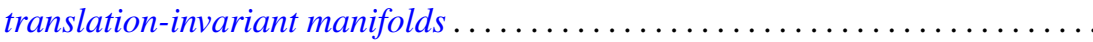

Erik Maurice Ellentuck, Infinite products of isols . . . . . . . . . . . . . . . . 49

William James Firey, Some applications of means of convex bodies . . . . . . . . 53

Haim Gaifman, Concerning measures on Boolean algebras ............. 61

Richard Carl Gilbert, Extremal spectral functions of a symmetric operator. . . . . . 75

Ronald Lewis Graham, On finite sums of reciprocals of distinct nth powers ..... 85

Hwa Suk Hahn, On the relative growth of differences of partition functions ...... 93

Isidore Isaac Hirschman, Jr., Extreme eigen values of Toeplitz forms associated

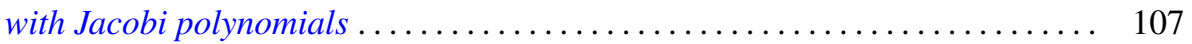

Chen-jung Hsu, Remarks on certain almost product spaces . . . . . . . . . . . 163

George Seth Innis, Jr., Some reproducing kernels for the unit disk . . . . . . . . . 177

Ronald Jacobowitz, Multiplicativity of the local Hilbert symbol . . . . . . . . . . . 187

Paul Joseph Kelly, On some mappings related to graphs ................. 191

William A. Kirk, On curvature of a metric space at a point . . . . . . . . . . . . 195

G. J. Kurowski, On the convergence of semi-discrete analytic functions . . . . . . . 199

Richard George Laatsch, Extensions of subadditive functions . . . . . . . . . . . 209

V. Marić, On some properties of solutions of $\Delta \psi+A\left(r^{2}\right) X \nabla \psi+C\left(r^{2}\right) \psi=0 \ldots 217$

William H. Mills, Polynomials with minimal value sets . . . . . . . . . . . 225

George James Minty, Jr., On the monotonicity of the gradient of a convex

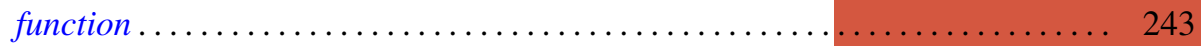

George James Minty, Jr., On the solvability of nonlinear functional equations of 'monotonic' type ................................... 249

J. B. Muskat, On the solvability of $x^{e} \equiv e(\bmod p) \ldots \ldots \ldots \ldots \ldots \ldots \ldots \ldots . \ldots \ldots$

Zeev Nehari, On an inequality of $P . R$. Bessack ................... 261

Raymond Moos Redheffer and Ernst Gabor Straus, Degenerate elliptic

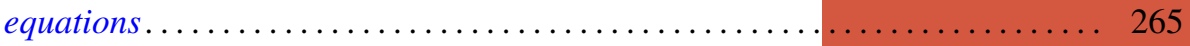

Abraham Robinson, On generalized limits and linear functionals . . . . . . . . . 269

Bernard W. Roos, On a class of singular second order differential equations with a

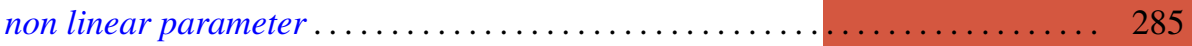

Tôru Saitô, Ordered completely regular semigroups . . . . . . . . . . . . . . . . 295

Edward Silverman, A problem of least area ....................... 309

Robert C. Sine, Spectral decomposition of a class of operators . . . . . . . . . 333

Jonathan Dean Swift, Chains and graphs of Ostrom planes . . . . . . . . . . . 353

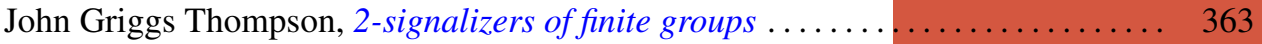

Harold Widom, On the spectrum of a Toeplitz operator . . . . . . . . . . . . . 365 